\title{
Disorder-Induced Effects in III-V Semiconductors with Mn
}

\begin{abstract}
J. MAŠEK AND F. MÁCA
Institute of Physics, AS CR, Na Slovance 2, 18221 Prague 8, Czech Republic

The substitution of $\mathrm{Mn}$ in the III-V diluted magnetic semiconductors leads to a strong electron scattering on impurities. Besides the features induced in the valence band by the hybridization with the Mn $d$-states, also the conduction band is affected by the absence of the Mn $s$-states at its edge. Also the high concentration of compensating donors modifies the band structure. This is shown on the absorption coefficient $\varepsilon_{2}(\omega)$ of GaP doped with $\mathrm{Mn}$ and Se. The absorption evaluated by ab initio density functional calculations starts with a smooth tail and does not show the structure typical of III-V materials. We analyze these features and the role of the donors on model systems using the tight-binding coherent potential approach.
\end{abstract}

PACS numbers: 71.15.Ap, 71.20.Nr, 71.55.Eq, 75.50.Pp

\section{Introduction}

The properties of the diluted magnetic semiconductors (DMS), such as II-VI and III-V materials doped with $\mathrm{Mn}$, are mostly determined by the presence of open $d$-shells of Mn. The $d$-electrons form the local magnetic moments, and the hybridization of the $d$-states with the band states leads to strong magnetooptical and magnetoelectric phenomena [1, 2]. In addition, the magnetic polarization of the band states of the host semiconductor results in kinetic exchange interaction between the moments.

The change of the crystal potential due to the Mn substitution has also an important direct effect on the band states. In the simplest description, the substitution is represented by replacing the atomic $s$ - and $p$-levels $E_{s}$ and $E_{p}$ of the host atom by the atomic levels of the impurity. In traditional II-VI DMS with $\mathrm{Mn}$, the differences in $E_{s}$ are of order of 1-2 eV [3]. This, with respect to the typical band structure of the II-VI semiconductors, represents a perturbation of an intermediate strength [4], which cannot be treated within the virtual crystal approximation (VCA). 
In the III-V semiconductors, however, the atomic levels of Mn differ from the atomic levels of the group-III cations even more. The change of $E_{s}$ in GaAs and $\mathrm{GaP}$ doped with $\mathrm{Mn}$ is approximately $4 \mathrm{eV}$ [3] and also the difference of the $p$-levels is remarkable. Strong impurity potential, comparable to the band width, is expected to cause a strong reconstruction of the electronic structure of the mixed crystal. This prediction of the tight-binding theory has been recently confirmed also by our recent calculations $[5,6]$.

We expect a particularly strong effect of the Mn substitution in the lowest conduction bands, because it is mostly composed of the cationic s-states. This means that the impurity scattering should have a pronounced implication on the near-edge absorption due to the violation of the momentum conservation and the appearance of the non-direct transitions [7] from the valence to the conduction band.

The situation is, however, more complex. It is well established that Mn doped III-V DMS reveal an almost complete compensation [8]. This means that the concentration of either intentional donors or native compensating defects increases proportionally to the concentration of $\mathrm{Mn}$ [6]. These donors represent an additional source of the disorder scattering.

We present here the first $a b$ initio study of the interband absorption in the III-V DMS. It is combined with tight-binding coherent potential approximation (CPA) calculations suitable for the interpretation of the disorder-induced features in the electronic structure of the dilute mixed crystals.

We concentrate on the interband absorption. That is why we consider only the limiting case of a complete compensation, where the IR transitions inside the valence band are absent.

\section{Absorption coefficient}

Although the main interest is paid to $(\mathrm{Ga}, \mathrm{Mn}) \mathrm{As}$, we applied our density functional study to $(\mathrm{Ga}, \mathrm{Mn}) \mathrm{P}$ with a wider band gap that is less sensitive to narrowing of the band gap due to the local density approximation. We take Sep as the compensating donor. The mixed crystal is represented by a periodic system with a large unit cell (LUC) containing 16 atoms. One molecular unit ( $\mathrm{Ga}-\mathrm{P}$ ) of the LUC is replaced by a close $\mathrm{Mn}-\mathrm{Se}$ pair. The electronic structure and the imaginary part of the dielectric function, $\varepsilon_{2}(\omega)$, were calculated by the full-potential linearized augmented-plane-waves (FPLAPW) method [9]. The spin-orbit coupling was not included in our spin-polarized calculations. The generalized gradient approximation to the exchange-correlation potential was used. The results are summarized in Figs. 1 and 2.

Figure 1 shows the spin-polarized total density of states (DOS) of the $\mathrm{Ga}_{7} \mathrm{MnP}_{7} \mathrm{Se}$ crystal in the low-temperature, ferromagnetic state altogether with the DOS of pure GaP. The bands of $\mathrm{Ga}_{7} \mathrm{MnP}_{7}$ Se are strongly magnetically polarized due to high concentration (12.5 at.\%) of magnetic impurities. The spin split- 


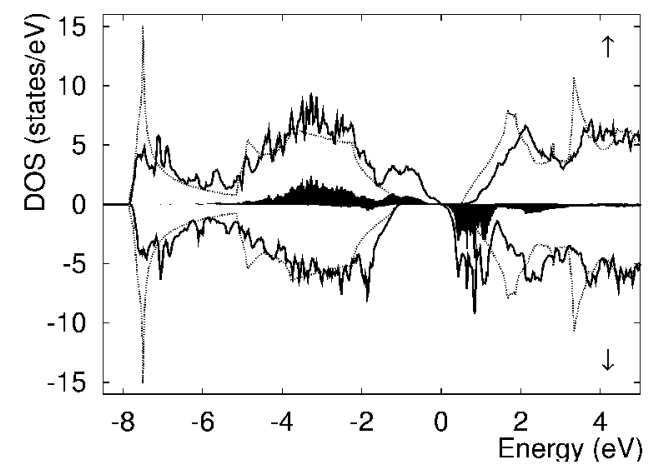

Fig. 1. Spin polarized density of states for $\mathrm{Ga}_{7} \mathrm{MnP}_{7} \mathrm{Se}$ (full line) compared to the rescaled DOS for pure GaP crystal (thin line). The hatched areas show the Mn $d$-states.

ting of the valence band $(1.2 \mathrm{eV})$ corresponds to the exchange constant $J_{p d} \approx 2 \mathrm{eV}$. The band gaps for both majority and minority spin electrons, $0.57 \mathrm{eV}$ and $1.29 \mathrm{eV}$, respectively, are smaller than the band gap obtained for the pure $\mathrm{GaP}(1.67 \mathrm{eV})$. In contrast to $(\mathrm{Ga}, \mathrm{Mn}) \mathrm{As}[6]$, the present material with a small overlap $(0.16 \mathrm{eV})$ of both band gaps is a semiconductor. The width of the common band gap is expected to increase for lower Mn doping.

Although the uppermost part of the majority spin DOS at the top of the valence band can be viewed as an impurity band formed by the acceptor states, no gap states are created due to Se impurities. They act as donors with zero activation energy. The presence of Se has no important effect, neither on the distribution of the Mn $d$-states (see Fig. 1) nor on the total DOS around the band gap. It means that most of the changes in the absorption should be attributed to $\mathrm{Mn}$. The $\mathrm{Mn}$ $s$-states contribute mostly to the broad maximum of the conduction band DOS around $4.5 \mathrm{eV}$ and they are almost completely absent at the edge of the conduction band, so that its bottom is locally eroded around Mn. Also the empty Mn $d$-states

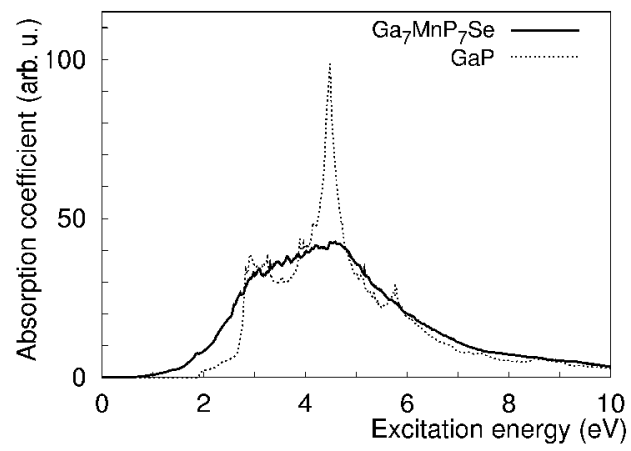

Fig. 2. Absorption coefficient, $\varepsilon_{2}(\omega)$, for $\mathrm{Ga}_{7} \mathrm{MnP}_{7} \mathrm{Se}$ (full line) compared to $\varepsilon_{2}(\omega)$ for pure GaP crystal (dotted line). 
substantially modify the nature of the bottom of the conduction band for the minority-spin electrons.

In Fig. 2 we compare the calculated $\varepsilon_{2}(\omega)$ for $\mathrm{Ga}_{7} \mathrm{MnP}_{7} \mathrm{Se}$ with the results for the GaP crystal from near-edge to the UV range. The absorption edge of pure $\mathrm{GaP}$ is followed by a steep increase in the absorption at $2.85 \mathrm{eV}$ ( $E_{1}$ transition) and by a main sharp peak at $4.4 \mathrm{eV}$ ( $E_{2}$ transition).

The change due to the $\mathrm{Mn}$ (and Se) doping is remarkable. The onset of the absorption is shifted to lower energies by $\approx 1.1 \mathrm{eV}$. Instead of well developed absorption edge, a smooth increase in the absorption reminds an Urbach tail [10]. The interband absorption does not show any structure typical of the zinc-blende semiconductors, only a broadened $E_{2}$ peak survives. The $E_{1}$ transition, more sensitive to the arrangement of the bands along the $A$ line in the Brillouin zone, disappears completely and the absorption gradually increases between $1.5 \mathrm{eV}$ and $4 \mathrm{eV}$.

\section{Spectral density}

To describe the DMS with lower impurity concentrations we use the coherent potential description [11] of the electronic structure of the DMS. The coherent potential method, resulting in configurationally averaged quantities, can also easily handle the room-temperature, magnetically disordered phase of the DMS. The averaging over all random distributions of the impurities restores the full translational symmetry of the lattice, so that the wave vector $k$ can be used as a quantum number. The spectral density $A(k, E)$, i.e. the DOS decomposed into contributions from the various points of the Brillouin zone, represents a detailed information about the dispersion of the electron states.

We apply a tight-binding version of the CPA [12] to GaAs co-doped with 4 at.\% of $\mathrm{Mn}$ and 4 at.\% of Se. The parametrization of the tight-binding Hamil-

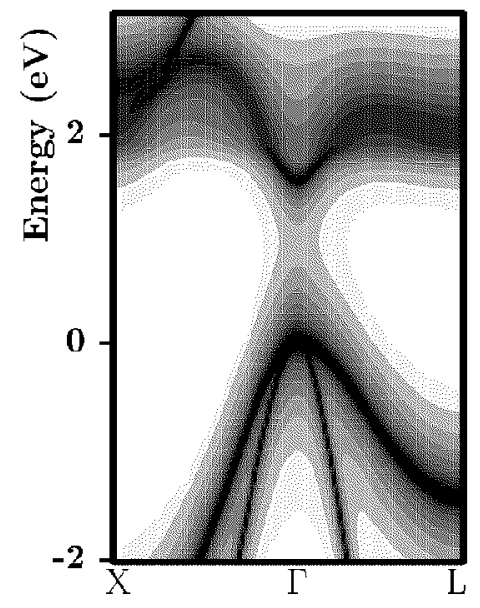

Fig. 3. Contour map of the spectral density $A(k, E)$ for the minority spin electrons in $\mathrm{Ga}_{0.96} \mathrm{Mn}_{0.04} \mathrm{P}_{0.96} \mathrm{Se}_{0.04}$ along the path $\mathrm{X}-\Gamma-L$ in the Brillouin zone. 
tonian provides a correct band gap for the pure GaAs crystal [13], as well as appropriate exchange splitting of the Mn $d$-states. As shown in Fig. 3 for minority spin electrons, the influence of the dopants on the spectral density is strong. Similar results were obtained for the majority spin electrons and also for the paramagnetic phase of $\mathrm{Ga}_{0.92} \mathrm{Mn}_{0.04} \mathrm{Se}_{0.04}$.

The linewidth of the Bloch states in the highest valence band and in the lowest conduction band is, in all cases, approximately $0.5 \mathrm{eV}$. The line width of the corresponding excitations, in particular of the $E_{1}$ transition is $\approx 1.0 \mathrm{eV}$, large enough to smear out the $E_{1}$ peak in $\varepsilon_{2}(\omega)$. However, it is important to note that the effect of the impurity broadening depends on the orbital composition of the bands and on the position in the Brillouin zone, as seen in Fig. 3. The narrow peaks of $A(k, E)$ indicate those parts of the bands where the effect of the impurities is rather weak.

\section{Comparison with other donors}

The almost complete compensation [8] in the III-V DMS with Mn means that the total concentration of donors, either intentional or native defects, is almost equal to the concentration of Mn, i.e. a few atomic percent. In such case, we have mixed rather than doped semiconductors and the modification of their band structure due to the donors must be considered. We compare the electronic structure of GaAs with 4 at.\% of $\mathrm{Mn}$ and with a corresponding concentration of the compensating donors. We consider donors in both anion and cation sublattices (Se, Sn), and As antisite defects.

The most favorite donors are the As antisite defects. They are double donors and create an impurity band deep in the band gap, centered at $0.8 \mathrm{eV}$ above the valence band. Also the co-doping with tin results in an impurity band around $1.7 \mathrm{eV}$, which is, however, resonant with the bottom of the conduction band. Both impurities give an inhomogeneous broadening $(\approx 0.7 \mathrm{eV})$ of the conduction-band states.

Finally, the intentional compensation with Se, considered in Secs. 2 and 3, can be viewed as an alloying of the III-V materials with MnSe, without formation of the impurity states.

\section{Summary}

The substitution of $\mathrm{Mn}$ in the GaAs crystal is connected with a scattering of electrons on the dopants. It results in broadening of the spectral lines and in smearing out the electron spectra. This is particularly seen in the calculated absorption coefficient. We found that the absorption edge of the compensated (Ga,Mn)P co-doped with Se is shifted by $\approx 1.0 \mathrm{eV}$ with respect to pure GaP crystal. The absorption is smooth, without any structure typical of the $E_{1}$ transitions in the III-V materials. 
Three aspects of the electron scattering on impurities can be distinguished: (i) Hybridization of the host states with the $\mathrm{Mn} d$-states, acting locally on the $p$-states of As atoms bonded to $\mathrm{Mn}$. This effect is particularly important in the upper part of the valence band. (ii) Impurity potential acting on the cationic sand $p$-states that modifies mostly the conduction band states. (iii) Scattering on compensating donors that are present in real III-V materials in a concentration comparable to the concentration of $\mathrm{Mn}$.

While (i) and (ii) are inherent to the Mn impurity, (iii) may be optimized by the choice of the compensating donor. We found that the effect of Se is weak in comparison to the influence of $\mathrm{Mn}$. The modification of the band states by both As antisite defects and substitutional $\mathrm{Sn}$ is much stronger. These two impurities also form impurity bands in (Ga,Mn)As while Se does not.

Assuming that the concentration of Mn determines the total number of the donors we deduce that the presence of the intentional donors can reduce the number of native antisite defects. If it is so, the co-doping of the III-V DMS with Se-like impurities will be quite important.

\section{Acknowledgment}

The financial support was provided by the Academy of Sciences of the Czech Republic (grant No. A1010214) and by RTN project "Computational Magnetoelectronics" of the European Commission (HPRN-CT-2000-00143).

\section{References}

[1] J.K. Furdyna, J. Appl. Phys. 64, R 29 (1988).

[2] H. Ohno, J. Magn. Magn. Mater. 200, 110 (1999).

[3] F. Herman, S. Skillman, Atomic Structure Calculations, Prentice-Hall, New Jersey 1963.

[4] B.E. Larson, K.C. Hass, H. Ehrenreich, A.E. Carlsson, Phys. Rev. B 37, 4137 (1988)

[5] J. Mašek, F. Máca, Acta Phys. Pol. A 100, 319 (2001).

[6] F. Máca, J. Mašek, Phys. Rev. B 65, 235209 (2002).

[7] W.E. Spicer, Phys. Rev. 154, 385 (1967).

[8] B. Beschoten, P.A. Crowell, I. Malajovich, D.D. Awschalom, F. Matsukura, A. Shen, H. Ohno, Phys. Rev. Lett. 83, 3073 (1999).

[9] P. Blaha, K. Schwarz, J. Luitz, WIEN97, FPLAPW package for calculating crystal properties, TU Vienna.

[10] F. Urbach, Phys. Rev. 92, 1324 (1953).

[11] B. Velický, S. Kirkpatrick, H. Ehrenreich, Phys. Rev. 175, 747 (1968).

[12] J. Mašek, B. Velický, V. Janiš, J. Phys. C 20, 59 (1987).

[13] J.N. Talwar, C.S. Ting, Phys. Rev. B 25, 2660 (1982). 